\title{
Immunization against the colonization factor antigen I of enterotoxigenic Escherichia coli by administration of a bivalent Salmonella typhimurium aroA strain
}

\author{
H.C.R. Guillobel ${ }^{1,2}$, \\ M.G. Luna ${ }^{1}$, \\ E.F. Camacho', \\ D.F. Almeida ${ }^{1}$ and \\ L.C.S. Ferreira ${ }^{1}$
}

\author{
'Laboratório de Fisiologia Celular, Instituto de Biofísica Carlos Chagas Filho, \\ Universidade Federal do Rio de Janeiro, Rio de Janeiro, RJ, Brasil \\ 2Departamento de Biofísica e Biometria, Instituto de Biologia, \\ Universidade do Estado do Rio de Janeiro, and Departamento de Genética, \\ Instituto de Biologia, Universidade Federal do Rio de Janeiro, Rio de Janeiro, \\ RJ, Brasil
}

\section{Correspondence \\ L.C.S. Ferreira \\ Laboratório de Fisiologia Celular Instituto de Biofísica Carlos Chagas Filho, CCS, UFRJ 21941-590 Rio de Janeiro, RJ Brasil \\ Fax: 55 (021) 280-8193 \\ E-mail: Icsf@ibccf.biof.ufrj.br \\ Research supported by PADCT and FINEP.}

Received May 6, 1997

Accepted January 16, 1998

\section{Abstract}

An expression plasmid (pCFA-1) carrying the $c f a B$ gene that codes for the enterotoxigenic Escherichia coli (ETEC) fimbrial adhesin colonization factor antigen I (CFA/I) subunit was constructed and used to transform a derivative of the attenuated Salmonella typhimurium aroA vaccine strain SL3261 carrying an F'lacI I. Treatment of the transformed strain with isopropyl-ß-D-thiogalactopyranoside (IPTG) resulted in elevated in vitro expression of the CFA/I subunit. Although flagellar function and lipopolysaccharide (LPS) synthesis were similar in both the parental and the recombinant strains, spleen colonization was reduced in the recombinant strain. All BALB/c mice parenterally inoculated with the recombinant strain developed significant antiCFA/I and anti-LPS serum antibody titers $(\mathrm{P}<0.05)$. Moreover, 2 of 5 mice orally inoculated with the engineered Salmonella strain developed anti-CFA/I intestinal $\operatorname{IgA}(\mathrm{P}>0.05)$ while $4 / 5$ of the same mice developed anti-LPS IgA $(\mathrm{P}<0.05)$. The results indicate that the vaccine strain elicited an antibody response against the bacterial host both after oral and intravenous immunization while the response against the CFA/I antigen was significant only after inoculation by the intravenous route.

\section{Introduction}

EnterotoxigenicEscherichia coli (ETEC) causes diarrheal disease with high morbidity and mortality rates among children in developing countries (1). It is also the infectious agent associated with traveller's diarrhea, which afflicts people travelling to places
Key words

- Salmonella typhimurium aroA

- Enterotoxigenic Escherichia coli

- Attenuated vaccines

- Colonization factor antigen I 
glycolipid receptors on the enterocyte surface. The CFA/I is one of the best characterized ETEC fimbriae and has a widespread occurrence in endemic areas, including South America (4-6). The CFA/I fimbriae contain a single protein subunit encoded by the plasmidial $c f a B$ gene endowed with structural and functional (adhesion) roles (7).

Effective immunization against ETECassociated diarrhea requires an anti-colonization mucosal IgA response (3). Studies with volunteers have shown that oral administration of large amounts of purified CFA/I fimbriae can induce protection against colonization by strains expressing CFA/I (8). However, protection afforded by purified CFA/I fimbriae is inefficient due to their degradation in the gastric and intestinal tracts (9). Alternatives to orally administered purified fimbrial preparations include direct delivery by orogastric instillation or the use of antigen-loaded biodegradable microspheres (10). However, difficult administration and/ or purification costs turned out to be serious obstacles for the use of anti-ETEC vaccinal strategies based on isolated CFA/I fimbriae. A distinct approach to achieve an immune response against ETEC fimbriae relies on the oral administration of inactivated ETEC strains expressing epidemiologically relevant fimbriae, i.e., CFA/I, CFA/II and CFA/IV (11). Although anti-CFA-specific protective immunity could be evoked with the inactivated cellular ETEC vaccine, quality control, safety and low immunogenicity represent relevant and unsolved issues which preclude the large scale use of such vaccine formulation. Therefore, new antigen delivery systems and/or alternative antigen administration routes for fimbrial adhesins are mandatory issues for the development of efficient ETEC vaccines.

Attenuated Salmonella strains can invade the surface of the intestinal mucosa and induce a strong immune response without causing any damage to the host $(12,13)$. Moreover, attenuated Salmonella strains can be used as vectors to deliver heterologous antigens of vaccine significance, thereby inducing cellmediated immunity as well as mucosal and serum antibody responses against the Salmonella vector and the passenger antigen. A large number of bivalentSalmonella-based vaccines against a variety of pathogens have been developed, expressing antigens of viruses, bacteria and parasites (14-16). Previous reports have also shown that attenuated Salmonella strains can express surface-exposed ETEC fimbriae, such as K88, K99 and CFA/I, from cloned sequences encompassing the complete encoding operons (17-19). However, expression of fimbrial subunits as an intracellular antigen by a Salmonella vaccine strain and the subsequent activation of the immune system by different administration routes has not been attempted.

In the present study we report an alternative strategy of intracellular CFA/I subunit expression based on the use of an attenuated S. typhimurium strain carrying a double-plasmid regulatory system. Oral and intravenous administration of the recombinant Salmonella vaccine strain resulted in serum and mucosal immune responses against the Salmonella vector and the fimbrial subunit.

\section{Material and Methods}

\section{Bacterial strains and plasmids}

The non-reverting aromatic-dependent (aroA) histidine-requiring Salmonella typhimurium SL3261 vaccine strain has been described previously (12) and was kindly provided by Dr. B.A.D. Stocker, Stanford University School of Medicine. The E. coli K-12 strains D1210 and DH5 $\alpha$ were employed in the selection of recombinant plasmid constructs. The ETEC strain TR 50/3 $\left(\mathrm{O} 63: \mathrm{H}^{-}, \mathrm{CFA} / \mathrm{I}^{+}, \mathrm{LT}^{+} / \mathrm{ST}^{+}\right)$was isolated from a diarrheic child in São Paulo and was kindly supplied by Dr. B.E.C. Guth, Escola Paulista de Medicina (6). The pKK223-3 plasmid was purchased from Pharmacia. The DH5 $\alpha$ 
F'IQ strain (GibcoBRL, Gaithersburg, MD, USA) was used as donor of F', a plasmid that carries the lacI $^{\mathrm{q}}$ gene and codes for kanamycin resistance.

\section{Media and growth conditions}

Bacteria were grown at $37^{\circ} \mathrm{C}$ with shaking. Luria-Bertani broth (LB) containing $1 \%$ tryptone, $0.5 \% \mathrm{NaCl}$, and $1 \%$ yeast extract was supplemented with $10 \mu \mathrm{g} / \mathrm{ml}$ of $2,3-$ dihydroxybenzoic acid (DHB, Sigma Chemical Co., St. Louis, MO). When appropriate, growth media were complemented with ampicillin $(100 \mu \mathrm{g} / \mathrm{ml})$, streptomycin $(70 \mu \mathrm{g} / \mathrm{ml})$, kanamycin $(25 \mu \mathrm{g} / \mathrm{ml})$, or rifampicin $(25 \mu \mathrm{g} /$ $\mathrm{ml})$. Spleen homogenates were plated onto brilliant green agar (BGA, Oxoid, Basingstoke, Hampshire, England) and xylose lysine deoxycholate agar (XLD, Oxoid) for detection of the Salmonella aroA strain, as previously described (20). Induction of $c f a B$ expression was obtained with isopropyl-B-Dthiogalactopyranoside (IPTG) (Sigma) at final concentrations ranging from 0.1 to 1 $\mathrm{mM}$. For evaluation of cell motility, Salmonella strains were grown on $0.35 \%$ agar plates containing $0.65 \%$ tryptone (21).

\section{Cloning of the $c f a B$ gene and construction of the CFA/l overexpressing plasmid}

Based on the available nucleotide sequence (22), forward (5'GGCCCGGGGGGAGGT CATTTCTAATGAAATTTAAAAAA ACT3') and reverse (5'GGCCCGGGTCAG GATCCCAA3') PCR primers were used to amplify the structural $c f a B$ gene, including a modified ribosome binding site, from purified total DNA extracted from the ETEC strain TR 50/3. ASmaI restriction site (underlined bases) was included in the 5 ' terminal of both primers. The PCR reactions were performed with Taq DNA polymerase using 30 temperature cycles of $1 \mathrm{~min}$ at $90^{\circ} \mathrm{C}, 1 \mathrm{~min}$ at $72^{\circ} \mathrm{C}$ and $1 \mathrm{~min}$ at $55^{\circ} \mathrm{C}$. A 550-bp DNA band corresponding to the $c f a B$ gene was purified from agarose gels, digested with $S m a \mathrm{I}$, and ligated to the $S m a \mathrm{I}$ cleaved pKK223-3 plasmid. The ligation mix was used to transform E. coli D1210 and DH5 $\alpha$ strains. Recombinant clones were selected by colony blotting using mouse antiCFA/I subunit-specific serum and rabbit antimouse IgG-peroxidase conjugate antibody (Sigma) after growth on LB plates containing ampicillin and $0.5 \mathrm{mM}$ IPTG. The recombinant plasmids were isolated and subjected to partial mapping with restriction enzymes. One plasmid, pCFA-1, with a correct insert size and proper orientation was chosen. The expression of the CFA/I subunit by the recombinant clones was determined by Western blot using CFA/I subunit-specific mouse antiserum and peroxidase conjugate antibody. Colony blots, plasmid DNA isolation, restriction enzyme digestion and ligation reactions were performed as described (23) or according to manufacturer instructions.

\section{Construction of the Salmonella vaccine strain}

F' $\operatorname{lac} I^{\mathrm{q}}$ was conjugally transferred to a rifampicin-resistant mutant of S. typhimurium SL3261 by overnight mating with DH5 $\alpha$ F'IQ on nitrocellulose filters (24). Transconjugants were selected on LB plates containing rifampicin and kanamycin at $37^{\circ} \mathrm{C}$. Exponential growth phase $S$. typhimurium cells carrying $\mathrm{F}^{\prime}$ lac $\mathrm{I}^{\mathrm{q}}$ were transformed with pCFA-1 by electroporation using a gene pulser apparatus (Bio Rad, Hercules, CA, USA) and conditions recommended by the manufacturers. Clones harboring both plasmids were selected on ampicillin-kanamycin-containing plates and the plasmid contents were evaluated by the alkaline lysis procedure and by agarose gel electrophoresis (23). The clone selected for further use was denominated HG3.

\section{Plasmid stability}

In vitro plasmid stability was measured in LB broth in the absence of antibiotic selection. 
IPTG $(0.5 \mathrm{mM})$ was added to an aerated exponential growth phase HG3 culture (optical density (OD) of 30 Klett units) and incubated at $37^{\circ} \mathrm{C}$. Samples were collected 4 and $24 \mathrm{~h}$ following IPTG addition, diluted in PBS and plated onto LB agar with or without ampicillin for viable cell counting.

\section{Vaccine preparation}

Exponential growth phase S. typhimurium HG3 and SL3261 strains were cultivated in LB containing ampicillin and streptomycin or streptomycin only, respectively. IPTG $(0.5 \mathrm{mM})$ was added to HG3 cultures (OD of 30 Klett units) and incubated at $37^{\circ} \mathrm{C}$ for $4 \mathrm{~h}$ in a water bath shaker. Cells were centrifuged, washed once with PBS, suspended in PBS containing 10\% glycerol to a final concentration of approximately $10^{10}$ colony-forming units $(\mathrm{cfu}) / \mathrm{ml}$ and kept at $-70^{\circ} \mathrm{C}$ until use.

\section{Immunization protocol and sample collection}

Five to 8 week-old male BALB/c mice were from the Instituto Nacional do Câncer (Rio de Janeiro). Bacterial cell suspensions were diluted in PBS and aliquots $(100 \mu \mathrm{l})$ containing approximately $10^{6} \mathrm{cfu}$ were administered intravenously (iv) via the tail vein to groups of 5 mice. The groups were immunized with a single dose on day 0 and boosted with an identical dose 30 days later. The animals were sacrificed 20 days after the booster dose. On the day before immunization and on the day of sacrifice blood samples were collected by retroorbital or tail bleeding. Serum samples were harvested and kept frozen at $-20^{\circ} \mathrm{C}$ until assayed. For oral immunization, groups of 5 mice were inoculated with $10^{9}$ cfu at days 0 and 4 followed by an identical booster dose 26 days later. Twenty days following the booster dose, the small intestine (the segment between the pylorus and the ileocecal junction) was excised, sliced and homogenized in $50 \mathrm{mM}$ EDTA and 0.1 $\mathrm{mg} / \mathrm{ml}$ phenylmethylsulfonyl fluoride (PMSF, Sigma). Samples were clarified by centrifugation, the supernatant was lyophilized, resuspended in $2 \mathrm{ml}$ of TEAN buffer (50 mM Tris-HCl, pH 7.5, 1 mM EDTA, 3 $\mathrm{mMNaN}_{3}$, and $200 \mathrm{mM} \mathrm{NaCl}$ ), and dialyzed overnight against TEAN buffer at $4^{\circ} \mathrm{C}$. Samples were kept frozen at $-20^{\circ} \mathrm{C}$.

\section{Sodium dodecyl sulfate-polyacrylamide gel electrophoresis (SDS-PAGE) and immunoblotting}

Whole-cell extracts were analyzed by SDS-PAGE followed by Coomassie brilliant blue R-250 staining. Mass-normalized cell samples (about $10^{7}$ exponentially growing cells) were suspended in electrophoresis sample buffer, boiled, and submitted to $15 \%$ PAGE (25). Proteins were transferred to nitrocellulose membranes and the CFA/I subunit was detected with mouse anti-CFA/I subunit serum and rabbit anti-mouse IgGperoxidase conjugate antibody as previously described (21). Bound antibodies were developed with the ECL chemoluminescence system (Amersham International plc., Little Chalfont, Buckinghamshire, England). Salmonella LPS was detected in proteinase Ktreated whole-cell extracts followed by silver staining of polyacrylamide gels (26).

\section{EUSA}

Serum and small intestine anti-CFA/I and anti-lipopolysaccharide (LPS) IgG responses were assayed on MaxiSorp microtiter plates (Nalge Nunc International, Naperville, IL, USA) previously coated with $0.1 \mu \mathrm{g} /$ well of purified and heat-dissociated CFA/I fimbriae or $0.2 \mu \mathrm{g} / \mathrm{well}$ of S. typhimurium LPS (Sigma). After overnight blocking with $1 \%$ skim milk in PBS at $4^{\circ} \mathrm{C}$, serum or gut homogenates, diluted in PBS-Tween $20(0.05 \%)$, were added to the wells and incubated at room temperature for $90 \mathrm{~min}$. Bound antibodies were detected with rabbit anti-mouse IgG- 
(for sera) or rabbit anti-mouse IgA- (for gut homogenates) peroxidase conjugate antibodies (Sigma) and developed with orthophenylenediamine (Sigma) and $\mathrm{H}_{2} \mathrm{O}_{2}$ as enzyme substrate. Absorbance was measured at 490 nm in a Bio Rad model 450 microplate reader. The titers are indicated by the absorbance at $490 \mathrm{~nm}$ of 1:6,400 (LPS) and 1:400 (CFA/I) dilutions of sera or 1:20 dilution of the gut homogenates.

\section{Spleen persistence of the Salmonella strains}

Six BALB/c mice were injected $i v$ with $10^{6} \mathrm{cfu}$ of HG3 or SL3261 strains. At days 1, 7 and 14 post-inoculation two animals of each group were sacrificed and the spleens removed. Spleens were homogenized separately in $1 \mathrm{ml}$ of cold $\mathrm{PBS}$ in a mechanical Potter apparatus and aliquots $(100 \mu \mathrm{l})$ plated onto BGA agar with and without ampicillin. When required spleen homogenates were diluted in PBS before plating. Plates were incubated overnight at $37^{\circ} \mathrm{C}$ for viable cell counts. Spleen samples were also spread on XLD agar plates to discriminate between the Salmonella aroA vaccine strain and wild type $S$. typhimurium contaminants. The aroA phenotype was detected by the translucent appearance of bacterial colonies on XLD

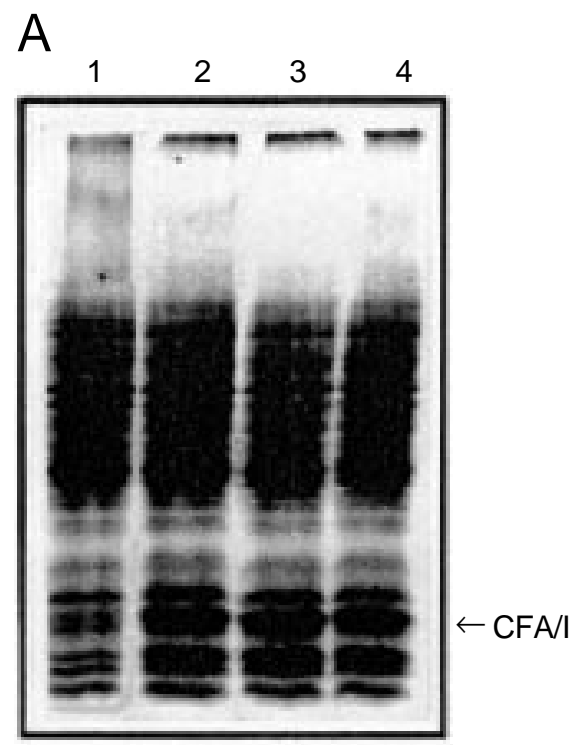

agar plates while colonies of wild type strains were dark due to $\mathrm{H}_{2} \mathrm{~S}$ production.

\section{Statistical analysis}

The standard deviation of the mean was calculated for all ELISA absorbance data and colony forming units (spleen persistence and plasmid stability). The mean of variously immunized groups of mice were compared by the Student $t$-test.

\section{Results}

\section{Construction of an S. typhimurium aroA vaccine strain expressing the CFA/I subunit}

Attempts to introduce pCFA-1 by electroporation into $S$. typhimurium SL3261 or $E$. coli DH5 $\alpha$ were unsuccessful, probably due to the toxic constitutive expression of the CFA/I subunit in the absence of the LacI repressor. Efficient repression of the $c f a B$ gene and successful transformation of Salmonella cells with pCFA-1 were obtained after conjugal transfer of F' lacI ${ }^{\mathrm{q}}$. The HG3 clone harboring both plasmids and expressing high inducible levels of the CFA/I subunit (about $5 \%$ of total protein) was selected for further use (Figure 1A). Since there was

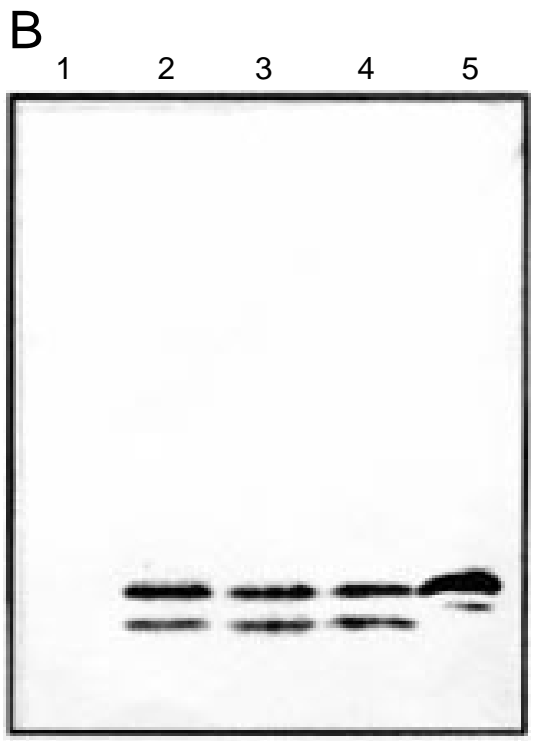

Figure 1 - Expression of the CFA/ I subunit by the recombinant $S$. typhimurium HG3 strain. Wholecell extracts (about $10^{7}$ cells) of the HG3 strain were subjected to SDS-polyacrylamide gel electrophoresis, stained with Coomassie blue $(20 \mu \mathrm{g} / \mathrm{well})(\mathrm{A})$, or submitted to Western blot analysis with anti-CFA/l subunit-specific serum diluted 1:2000 (B). Lane 1: non-induced HG3 cells; lanes 2, 3 and 4: HG3 cells induced with $0.1 \mathrm{mM}, 0.5 \mathrm{mM}$ and 1 mM IPTG, respectively; lane 5: $5 \mu \mathrm{g}$ of purified CFA/l subunit. 
Figure 2 - Lipopolysaccharide composition of $S$. typhimurium SL3261 and HG3 strains. Bacteria grown in LB (about $10^{7}$ cells) were treated with proteinase $\mathrm{K}$ and subjected to SDS-PAGE, followed by silver staining of LPS. Lane 1 - S. typhimurium SL3261; lane 2 - $S$. typhimurium HG3. no significant difference in the expression of the CFA/I subunit in cells induced by IPTG concentrations ranging from $0.1 \mathrm{mM}$ to 1 $\mathrm{mM}$, the concentration of $0.5 \mathrm{mM}$ was chosen for further experiments (Figure 1A). The recombinant and native $\mathrm{CFA} / \mathrm{I}$ subunits have similar electrophoretic mobilities with an apparent molecular weight of $15 \mathrm{kDa}$. One band of approximately $14 \mathrm{kDa}$, a probable degradation product, was observed in wholecell extracts of IPTG-induced HG3 cultures and in purified CFA/I subunit preparations (Figure 1B). Immunoblots of subcellular fractions of IPTG-induced HG3 cells showed that the recombinant CFA/I subunit was found predominantly in the soluble fraction (cytoplasm and periplasm) of sonically disrupted cells (data not shown).

\section{Phenotypic properties and plasmid stability of the S. typhimurium HG3 strain}

No modification in LPS $\mathrm{O}$ antigen side chains of the HG3 strain could be detected in silver-stained polyacrylamide gels of pro-

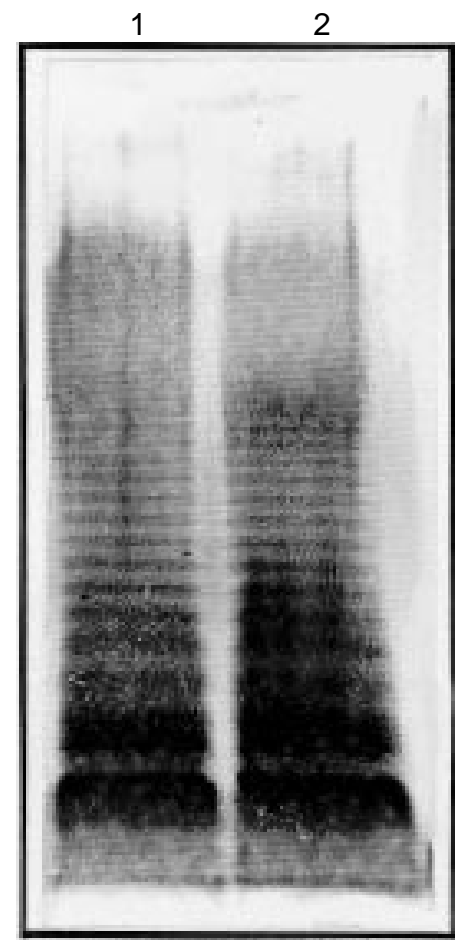

teinase $\mathrm{K}$ whole-cell digests (Figure 2). Similarly, no significant impairment of HG3 strain motility was detected in relation to the parental SL3261 strain, as evaluated by the size of swarming halos formed on soft agar plates after overnight growth (data not shown).

In the absence of ampicillin and IPTG there was no significant plasmid segregation during in vitro growth of the HG3 strain. All colonies tested after $24 \mathrm{~h}$ growth in LB medium were still resistant to ampicillin and were able to express the CFA/I protein when IPTG was added (data not shown). On the other hand, incubation of HG3 cells in the presence of $0.5 \mathrm{mM}$ IPTG for $4 \mathrm{~h}$ resulted in a significant reduction (approximately 40\%) of ampicillin-resistant cells which indicated segregation of the recombinant plasmid (Table 1). The number of ampicillin-sensitive colonies was increased to approximately $80 \%$ of the total counts when the incubation time was extended to $24 \mathrm{~h}$ (Table 1). As expected, only ampicillin-resistant colonies were able to express the CFA/I subunit when exposed to IPTG (data not shown).

\section{Persistence and plasmid stability of the S. typhimurium HG3 strain during in vivogrowth}

The number of Salmonella organisms per spleen in animals which received a single $i v$ dose of $10^{6} \mathrm{cfu}$ ranged from $1,000 \mathrm{cfu}$ in those inoculated with HG3 to >10,000 cfu in those inoculated with SL3261 one week after injection (Figure 3). In both groups there was a slight increase in the number of Salmonella organisms per spleen two weeks after injection (Figure 3). Both strains were cleared from the spleens of inoculated mice one month after inoculation and all animals were apparently healthy at sacrifice (data not shown).

\section{Immunization of mice with the S. typhimurium HG3 strain}

All mice inoculated $i v$ with either HG3 or 
Table 1 - Evaluation of pCFA-1 stability in HG3 cells grown under inducing conditions.

aExponential phase HG3 cells were incubated in the presence of $0.5 \mathrm{mM}$ IPTG for 4 or $24 \mathrm{~h}$. bResults are reported as the mean \pm SD of quadruplicates from two identical experiments.

\begin{tabular}{lcc}
\hline $\begin{array}{l}\text { Inducing } \\
\text { condition }\end{array}$ & \multicolumn{2}{c}{ Number of viable cells $(\mathrm{cfu} / \mathrm{ml})^{\mathrm{b}}$} \\
\cline { 2 - 3 } & LB & LB + ampicillin \\
\hline- & $4.75 \pm 0.20 \times 10^{9}$ & $4.52 \pm 0.27 \times 10^{9}$ \\
$4 \mathrm{~h}$ & $3.15 \pm 0.20 \times 10^{8}$ & $1.91 \pm 0.20 \times 10^{8}$ \\
$24 \mathrm{~h}$ & $1.55 \pm 0.14 \times 10^{9}$ & $3.70 \pm 0.80 \times 10^{8}$
\end{tabular}

SL3261 had significant $(\mathrm{P}<0.05)$ serum antiLPS IgG titers while non-immunized mice sera showed background ELISA values (Figure 4A). Similarly, mice immunized with HG3, but not those immunized with SL3261, had elevated $(\mathrm{P}<0.05)$ serum anti-CFA/I IgG levels while no significant reaction was found in the sera of non-immunized mice (Figure 4B).

Four of 5 mice orally immunized with HG3 had significant $(\mathrm{P}<0.05)$ anti-LPS IgA levels in gut homogenates whereas 5 of 5 mice immunized with SL3261 elicited an anti-LPS IgA response $(\mathrm{P}<0.05)$ (Figure $5 \mathrm{~A}$ ) and 2 of 5 mice immunized with HG3 showed an anti-CFA/I IgA response in gut homogenates $(\mathrm{P}<0.10)$ (Figure 5B). In contrast, orally administered SL3261 failed to elicit an antiCFA/I IgA response (Figure 5B). Similarly, no anti-CFA/I IgA response was detected in a serum pool from non-immunized mice. These results indicate that the HG3 vaccine strain could elicit an antibody response against the host antigen after both oral and intravenous immunization while the response against CFA/I was significant only after intravenous inoculation.

\section{Discussion}

The use of attenuated Salmonella strains as vehicles to deliver heterologous antigens

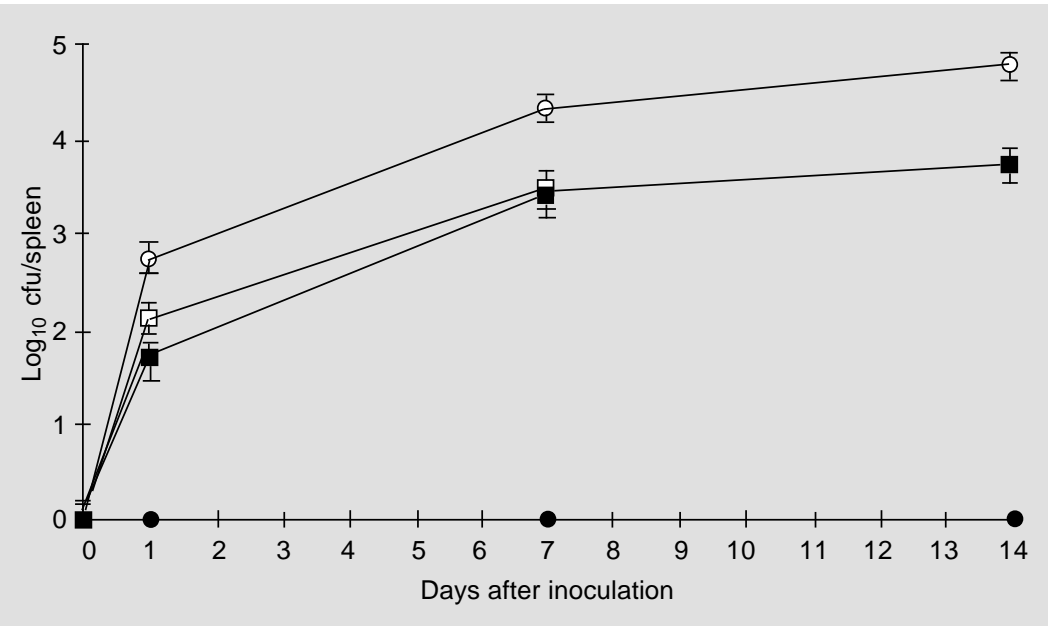

Figure 3 - Spleen persistence of $S$. typhimurium SL3261 and HG3 strains. BALB/c mice received a single iv dose of $10^{6} \mathrm{cfu}$ of SL3261 (circles) or $10^{6} \mathrm{cfu}$ of HG3 (squares). Mice were sacrificed and spleens removed at the times indicated. Spleen homogenates were plated onto BGA with (closed symbols) or without (open symbols) ampicillin at $100 \mu \mathrm{g} / \mathrm{ml}$. Results are reported as the mean \pm SD of quadruplicates from two identical experiments.

to the immune system represented an important step in the generation of orally administered bivalent vaccines (15). However, this approach faces two main difficulties. First, it is assumed that large initial amounts of the heterologous antigen should be accumulated by the vaccine strain in order to prime the host immune system, and second, sustained expression of the antigen seems to be required during the persistence time of the strain in host tissues $(14,16,27)$. Plasmid expression is usually employed to attain high antigen levels but the metabolic overload or toxicity associated with excessive accumulation of the heterologous antigen may be deleterious to the vaccine strain. In the present study, for instance, the constitutive expression of the $c f a B$ gene under the control of a strong promoter was lethal to the bacterial cell. The use of a double-plasmid regulatory system composed of a tac promotorcontrolled $c f a B$ gene-encoding plasmid and a second plasmid encoding the LacI repressor allowed the generation of a high antigen load without seriously affecting the viability of the strain or the expression of phenotypic properties relevant for the immunogenic potency. The presence of the recombinant plas- 
Figure 4 - Serum antibody response of mice immunized iv with $S$. typhimurium HG3 cells. The serum samples of 5 mice immunized with $10^{9} \mathrm{cfu}$ of HG3 were analyzed individually (numbers 1 to 5) by IgG-ELISA for Salmonella $\mathrm{LPS}$ at a final dilution of $1: 6,400(A)$ and the CFA/I subunit at a final dilution of $1: 400$ (B). Results obtained with pools of non-immunized (NI) and SL3261-inoculated (SL3261) mouse sera are also shown. Results are reported as the mean \pm SD of quadruplicate wells of two identical experiments.
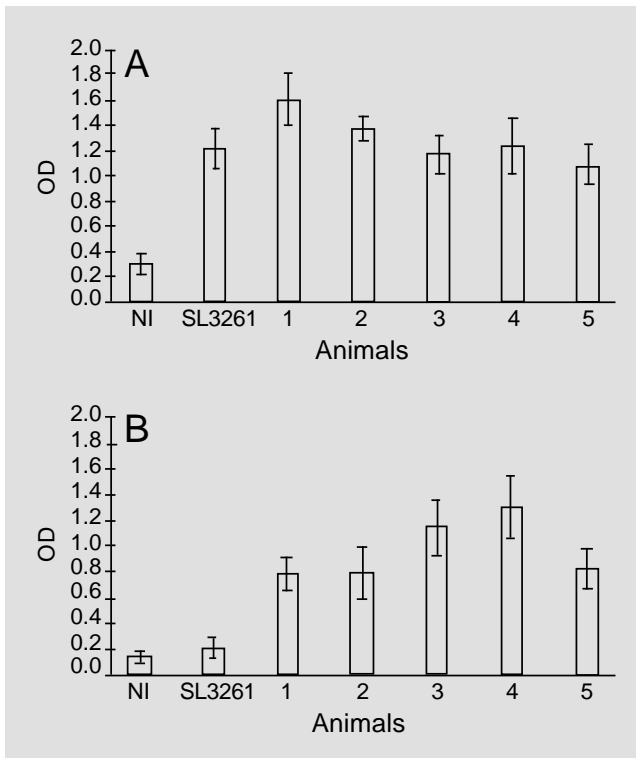

Figure 5 - Secreted antibody response of mice orally immunized with S. typhimurium HG3 cells. The gut homogenates of 5 mice immunized with $10^{9} \mathrm{cfu}$ of HG3 were analyzed individually (numbers 1 to 5 ) by IgA-ELISA for Salmonella LPS (A) and the CFA I subunit (B). Results obtained with pools of non-immunized (NI) and SL3261-inoculated (SL3261) mouse gut homogenates are also shown. All samples were diluted 1:20 in PBS before testing. Results are reported as the mean $\pm S D$ of quadruplicate wells for two identical experiments.
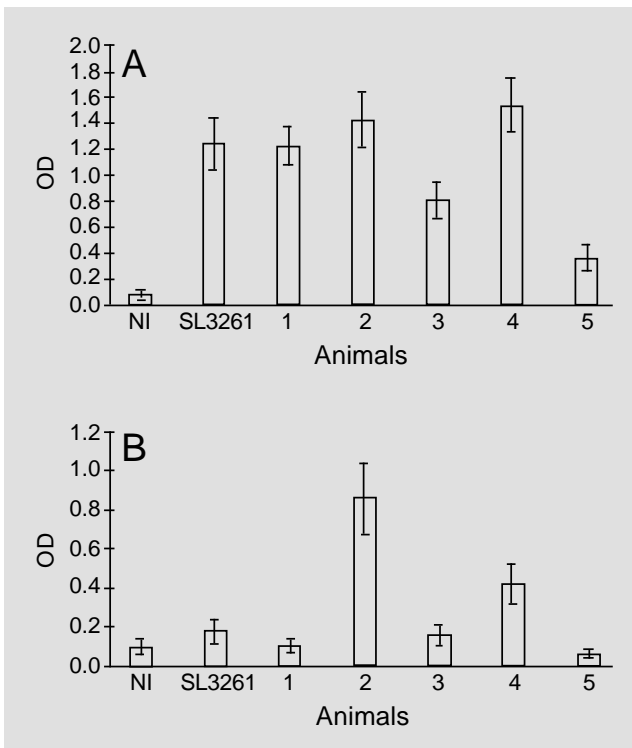

mid did not inhibit the ability of the Salmonella strain to persist in spleens of inoculated mice and, more relevant, the present data show that the activation of systemic and secretory immune responses could be triggered in $i v$ and orally inoculated mice, respectively.

CFA/I fimbria expression in ETEC cells is a rather complex process and depends on environmental factors such as composition of the growth medium, temperature and growth stage of the cells. This fact is reflected by the requirement of at least 5 genes acting at the transcriptional or post-transcriptional level for expression of the $c f a B$ gene and assemblage of CFA/I fimbriae (28). Therefore, instead of using fimbriated cells generated by the introduction of the whole CFA/I operon in the Salmonella strain, we cloned and expressed the CFA/I subunit structural gene under control of a strong inducible promoter leading to high intracellular levels of the antigen. The expression of cell-associated unpolymerized CFA/I subunits by the Salmonella vaccine strain may be advantageous for the induction of systemic and secreted antibodies since the antigen is protected from the harsh gastric and enteric environments which can degrade surfaceexposed fimbriae. Moreover, elevated levels of CFA/I subunit could be easily obtained and controlled during in vitro growth of the vaccine strain, permitting a better standardization of the antigen load. Finally, dissociated CFA/I subunits, but not intact fimbriae, expose immunorecessive epitopes, which confer cross-reaction with other ETEC fimbriae such as CS1, CS2, CS4, CS17 (29-31). Taking into account all of these facts, the ability of the present HG3 vaccine strain to induce anti-CFA/I subunit-specific antibodies may represent an important step toward the generation of a broad-range anti-colonization ETEC vaccine.

It has been suggested that the initial load rather than the antigen synthesized during persistence of the bacterial cells in the host tissues is the most critical event for priming the gut-associated lymphoreticular tissue (GALT) and activation of the host immune response against the heterologous antigen delivered by orally administered recombinant Salmonella vaccine strains (27). The development of the S. typhimurium HG3 strain allowed us to obtain high initial CFA/ I subunit levels but presumably the recombinant bacteria could not express additional amounts of the heterologous antigen after 
administration to the host. Therefore, expression of the heterologous antigen in host tissues was apparently not essential for the activation of the systemic response since all mice immunized $i v$ with the HG3 strain showed elevated anti-CFA/I subunit-specific IgG titers. In contrast, only 2 of 5 mice orally immunized with the HG3 strain developed detectable anti-CFA/I subunit-specific secreted IgA levels in their guts. The lack of a more efficient mucosal immune response may reflect the lack of in vivo CFA/I subunit expression by the Salmonella cells colonizing the Peyer's patches of vaccinated mice. Future experiments should address the question of how the priming and subsequent colonization of the mucosal immune system by the recombinant HG3 strain could contribute to the activation of a secretory immune response. Further improvements of the activation of the mucosal immune response may require continuous low level expression of the heterologous antigen or co-administra- tion of a mucosal adjuvant.

Since the initial observations of Yamamoto et al. (32), surface expression of intact CFA/I fimbriae by recombinant $\mathrm{Sal}$ monella vaccine strains has represented one possible way to raise a specific immune response against the ETEC adhesin $(17,19)$. The present results show that intracellular expression of CFA/I subunits by an attenuated Salmonella strain is an alternative approach to the problem of antigen presentation which may become a new strategy for the development of anti-Salmonella/ETEC bivalent vaccines.

\section{Acknowledgments}

We thank Celso Pereira, Joana Iabrudi Carinhanha and Kelen Carine Costa Soares for technical assistance, and B.A.D. Stocker and B.E.C. Guth for supplying the bacterial strains.

\section{References}

1. Sack RB (1975). Human diarrheal disease caused by enterotoxigenic Escherichia coli. Annual Review of Pathogenesis, 29: 333-353.

2. Levine MM (1987). Escherichia coli that cause diarrhea: enterotoxigenic, enteropathogenic, enteroinvasive, enterohemorrhagic, and enteroadherent. Journal of Infectious Diseases, 155: 377-389.

3. Levine MM, Kaper JB, Black RE \& Clements ML (1983). New knowledge on pathogenesis of bacterial enteric infections as applied to vaccine development. Microbiological Reviews, 47: 510-550.

4. Evans DG, Silver RP, Evans Jr DJ, Chase DG \& Gorbach SL (1975). Plasmid controlled colonization factor associated with virulence in Escherichia coli enterotoxigenic for humans. Infection and Immunity, 12: 656-667.

5. Binsztein N, Jouve MJ, Viboud GI, Moral LL, Rivas M, Orskov I, Åhrén C \& Svennerholm A-M (1991). Colonization factors of enterotoxigenic Escherichia coli isolated from children with diarrhea in Argentina. Journal of Clinical Microbiology, 29: 1893-1898.
6. Guth BEC, Aguiar EG, Griffin PM, Ramos SRTS \& Gomes TAT (1994). Prevalence of colonization factor antigens (CFAs) and adherence to HeLa cells in enterotoxigenic Escherichia coli isolated from feces of children in São Paulo. Microbiology and Immunology, 38: 695-701.

7. Bühler T, Hoschützky H \& Jann K (1991). Analysis of colonization factor antigen I, an adhesin of enterotoxigenic Escherichia coli O78:H11: fimbrial morphology and location of the receptor-binding site. Infection and Immunity, 59: 3876-3882.

8. Evans DG, Graham DY, Evans Jr DJ \& Opekun A (1984). Administration of purified colonization factor antigens (CFA/l, CFA/II) of enterotoxigenic Escherichia coli to volunteers. Gastroenterology, 87: 934940.

9. Schmidt M, Kelley EP, Tseng LY \& Boedecker EC (1985). Towards an oral $E$. coli pilus vaccine for traveller's diarrhea: susceptibility to proteolytic digestion. Gastroenterology, 82: 1575-1582.

10. Edelman R, Russel RG, Losonsky G, Tall BD, Tacket CO, Levine MM \& Lewis DH (1993). Immunization of rabbits with en- terotoxigenic E. colicolonization factor antigen (CFA/I) encapsulated in biodegradable microspheres of poly (lactide-coglycolide). Vaccine, 11: 155-158.

11. Holmgren J \& Svennerholm A-M (1996). Oral vaccines against cholera and enterotoxigenic Escherichia coli diarrhea. In: Kiyono H, Ogra PL \& McGhee JR (Editors), Mucosal Vaccines. Academic Press, San Diego, CA.

12. Hoiseth SK \& Stocker BAD (1981). Aromatic-dependent Salmonella typhimurium are non-virulent and effective as live vaccines. Nature, 291: 238-239.

13. Dougan G, Chatfield S, Pickard D, O'Callaghan D \& Maskell D (1988). Construction and characterization of Salmonella vaccine strains harbouring mutations in two different aro genes. Journal of Infectious Diseases, 158: 1329-1335.

14. Walker RI (1994). New strategies for using mucosal vaccination to achieve more effective immunization. Vaccine, 12: 387400.

15. Fairweather NF, Chatfield SN, Makoff AJ, Strugnell RA, Bester J, Maskell DJ \& Dougan $G$ (1990). Oral vaccination of mice 
against tetanus by use of a live attenuated Salmonella carrier. Infection and Immunity, 58: 1323-1326.

16. Walker MJ, Rohde M, Timmis KN \& Guzmán CA (1992). Specific lung mucosal and systemic immune responses after oral immunization of mice with Salmonella typhimurium aroA, Salmonella typhi Ty21a, and invasive Escherichia coli expressing recombinant pertussis toxin $\mathrm{S} 1$ subunit. Infection and Immunity, 60: 4260-4268.

17. Giron JA, Xu J-G, Gonzalez CR, Hone D, Kaper JB \& Levine MM (1995). Simultaneous expression of CFA/I and CS3 colonization factor antigens of enterotoxigenic Escherichia coli by $\triangle$ aroC, $\triangle$ aroD Salmonella typhi vaccine strain CVD 908. Vaccine, 13: 939-946.

18. Morona R, Morona JK, Considine A, Hackett JA, van den Bosch L, Beyer L \& Attridge SR (1994). Construction of K88and K99-expressing clones of Salmonella typhimurium G30: immunogenicity following oral administration to pigs. Vaccine, 12: 513-517.

19. Wu S, Pascual DW, VanCott JL, McGhee JR, Maneval Jr DR, Levine MM \& Hone DM (1995). Immune response to novel Escherichia coli and Salmonella typhimurium vectors that express colonization factor antigen I (CFA/l) of enterotoxigenic $E$. coli in the absence of the CFA/l positive regulator cfaR. Infection and Immunity, 63: 4933-4938.

20. Cooper GL, Venables LM, Woodward MJ
\& Hormaeche CE (1994). Vaccination of chickens with strain CVL30, a genetically defined Salmonella enteritidis aroA live oral vaccine candidate. Infection and Immunity, 62: 4747-4754.

21. Luna MG, Martins MM, Newton SMC, Costa SOP, Almeida DF \& Ferreira LCS (1997). Cloning and expression of colonization factor antigen I (CFA/I) epitopes of enterotoxigenic Escherichia coli (ETEC) in Salmonella flagellin. Research in Microbiology, 148: 217-228.

22. Karjalainen TK, Evans DG, So M \& Lee C$H$ (1989). Molecular cloning and nucleotide sequence of the colonization factor antigen I gene of Escherichia coli. Infection and Immunity, 57: 1126-1130.

23. Sambrook J, Fritsch EF \& Maniatis T (1989). Molecular Cloning: A Laboratory Manual. 2nd edn. Cold Spring Harbor Laboratory Press, Cold Spring Harbor, NY.

24. Miller JH (1992). A Short Course in Bacterial Genetics: a Laboratory Manual and Handbook for Escherichia coli and Related Bacteria. Cold Spring Harbor Laboratory Press, Los Angeles, CA.

25. Laemmli UK (1970). Cleavage of structural proteins during the assembly of the head of the bacteriophage T4. Nature, 227: 680-685.

26. Hitchcock PJ \& Brown TM (1983). Morphological heterogeneity among Salmonella lipopolysaccharide chemotypes in silver-stained polyacrylamide gels. Journal of Bacteriology, 154: 269-277.

27. Cárdenas L, Dasgupta U \& Clements JD
(1994). Influence of strain viability and antigen dose on the use of attenuated mutants of Salmonella as vaccine carriers. Vaccine, 12: 833-840.

28. Savelkoul PHM, Willshaw GA, McConnell MM, Smith HR, Hamers AM, van der Zeijst BAM \& Gaastra W (1990). Expression of CFA/l fimbriae is positively regulated. Microbial Pathogenesis, 8: 91-99.

29. Rudin A, McConnell MM \& Svennerholm A-M (1994). Monoclonal antibodies against enterotoxigenic Escherichia coli colonization factor antigen I (CFA/I) that cross-react immunologically with heterologous CFAs. Infection and Immunity, 62: 4339-4346.

30. Rudin A, Olbe L \& Svennerholm A-M (1996). Monoclonal antibodies against fimbrial subunits of colonization factor antigen I (CFA/I) inhibit binding to human enterocytes and protect against enterotoxigenic Escherichia coli expressing heterologous colonization factors. Microbial Pathogenesis, 20: 35-45.

31. Rudin A \& Svennerholm A-M (1996). Identification of a cross-reactive continuous Bcell epitope in enterotoxigenic Escherichia coli colonization factor antigen I. Infection and Immunity, 64: 4508-4513.

32. Yamamoto $T$, Tamura $Y$ \& Yokita $T$ (1985). Enteroadhesion fimbriae and enterotoxin of Escherichia coli: genetic transfer to a streptomycin-resistant mutant of the galE oral-route live-vaccine Salmonella typh Ty21a. Infection and Immunity, 50: 925928. 